\title{
2d set
}

QL.

461

S67

1894

ENT
SOCIETA EITCIOLOGICA ITALIANA

Statuto, 1894 
te. 


\section{4}

\section{SOCIETA ENTOMOLOGICA ITALIANA}

\section{S T A T U T O}

ELEYCO DEI SOCI E DEGLI ASSOCIITI - ELEXCO DELLE SOCIETI

E DELLE AGCAUEHE CORRSPONUENTI

FIRENZE

TIPOARA FIA M. R I C C I

Via S.ı Gallo, 31

$\overline{1594}$ 



\section{4}

\section{SOCIETÁ ENTOMOLOGICA ITALIANA}

\section{S T A T U T O}

ELEVGO IIEI SOCI E DEGLI ISSOCIITI - ELEVCO DELLE SOCIETI

E IIELLE IGCGIHEVIE CORHISPO YIEVTI

FIRENZE

T I P O G R A F I A Mr. R I C C I

Via S:n Gallo, 31

$\overline{1894}$ 


\section{STATUTO}

\section{SOCIETA ENTOHOLOGICA ITALIANA}

CON LE MODIFICAZIONI DFLLHERATE

nelle Adunanze Generali del 6 Aprile 1873, del 2 Giuǵno 1887, del 12 Maǵǵio 1882, del 30 Giuǵno 1889 e del 4 Febbraio 1894.

Titolo Priso.

\section{Costituzione alellat societio.}

Arr. 1. - Sotto il titolo di

\section{SOCIETẢ ENTOMOLOGICA ITALIANA}

è istituita, con sede in Firenze, un'associazione scientifica, la quale lia per iscopo di cooperare al progresso della entomologia, si pura come applicata, e, pigliando specialmente di mira gl'insetti e gli artropodi della fanna italiana, di promuoverne lo studio da ogni punto di vista, tanto in ordine alle scienze, quanto agli utili che se ne possono ricavare per l'economia domestica, per la salute degli uomini e degli animali, per l'agricoltura e per le arti indnstriali.

Алт. 2. - Per conseguire questo fine, essa tiene adunanze, stabilisce e dirige escursioni, e pubblica un giornale col titolo di Bullettino della Societì Entomologica Italiana.

Art. 3. - Si compone di un numero illimitato di Soci. - Gli italiani e gli stranieri possono egualmente appartenervi.

Arт. 4. - Un Consiglio generale, dal cui seno vengono tratti gli ufficiali per comporre un Comitato residente, la rappresenta e ne cura l'amministrazione, secondo le norme che saranno indicate nel Titolo terzo. 


\section{Titolo Secondo.}

\section{Dei Soei.}

Arт. 5. - La qualita di Socio si acquista in virti di una deliberazione presa dal Consiglio generale, o dal Comitato residente, sulla proposta di dne persone già ascritte alla Società.

Art. 6. - I Soci sono di due categorie: Soci effettivi e Soci studenti. I primi pagano una tassa annua di lire quindici (15), i secondi di lire dieci (10). I soci studenti non possono rimanere tali per più di 5 anni, dopo di che direntano soci effettivi e pagano L. 15.

La quota annule tanto dei Soci effettivi che studenti è dovuta nel primo trimestre dell'amno. I Soci effettivi che pagheranno in una sola volta lire duecento (200) saramno Soci perpetui. I nuovi Soci, appena pagata la tassa, hanno diritto al diploma di nomina senza aumento di spesa.

Arr. 7. - Le ammissioni dei Soci sia effettivi o sturdenti vengono sempre considerate, in ordine alla tassa e al diploma, come fatte il $10^{0}$ di gemaio dell'anno in corso.

ArT. 8. - Ogni Socio effettivo o studente ha diritto, nell'anno in cui comincia a decorrere la sua tassa, di ricevere gratuitamente una copia di tutte le pubblicazioni sociali dell'anno stesso, e d'intervenire alle admanze ed alle escursioni di studio promosse e dirette dalla Società.

Arт. 9. - $\dot{\mathrm{E}}$ in facoltà dei Soci di pagare una tassa doppia, tripla, quadrupla, ecc. di quella indicata all'art. 6: nel qual caso essi acquisteranno diritto a ricevere due, tre, quattro, ecc. copie di tutte le pubblicazioni sociali, mentre non avranno però che un sol voto nelle admnanze.

ART. 10. - Coloro che per un intero anno mancassero al pagamento della tassa, saranno, con lettera del Tesoriere, invitati a mettersi in pari; lo che non facendo entro il primo trimestre dell'anno segrnente, potranno per deliberazione del Comitato residente, esser cancellati dall'albo della Società.

Titolo Terzo.

\section{Del Conniglio Genenale e del Comitato Residente.}

Art. 11. - Il Consiglio generale si compone di Un Presidente - Tre Vice-Presidenti 
Un Segretario degli Atti - Un Segretario del Carteggio Un Tesoriere - Dodici Consiglieri.

Uno dei Consiglieri disimpegna le funzioni di Archivista. Bibliotecario.

ArT. 12. - I titolari a questi uffici vengono eletti dai soci rimiti in admunza generale, per mezzo di schede segrete, ed a maggioranza di voti.

Occorrendo votazioni di ballottaggio, si procederà ad esse, seduta stante.

Esaurita la rotazione, quando l'uguaglianza di voti tra i due candidati fosse mantenuta, la sorte decide della elezione.

I Soci potranno in occasione dell'indicata elezione del Consiglio, farsi rappresentare da altri Soci, o spedire in schede sigillate i nomi delle persone da essi designate per le diverse cariche.

Ant. 13, - Il Presidente, un Vice-Presidente, i Segretari, il Tesoriere e due Consiglieri debbono essere scelti tra $\mathrm{i}$ Soci che hanno domicilio in Firenze, o in luogo ricino. Gli altri membri del Consiglio (ciò due Vice-Presiclenti e 10 Consiglieri) possono aver residenza in qualunque parte d'Italia.

Art. 14. - Ogni anno escono di carica un Vice-Presidente e quattro Consiglieri. Il Presidente, i Segretari e il Tesoriere restano in Ufficio per tre anni. Tutti sono rieleggibili.

Arr. 15. - Il Consiglio generale rappresenta la Societa di cui ha l'alta direzione. Alla fine dell' anno discute ed approxa il bilancio preventivo per l'amno seguente, e trasmette entro il primo trimestre il bilancio consuntivo ai Sindaci, che dopo averlo esaminato lo presentano alla Società nella Adunanza generale di cui all'art. 24.

I Sindaci vengono eletti come è stato indicato piu sopra per i membri del Consiglio, e rimangono in carica un anno soltanto.

Art. 16. - Il Presidente, un Vice-Presidente, i Segretari. il 'Tesoriere e due Consiglieri compongono il Comitato residente, a cui è afficlata l'Amministrazione sociale, liu compilazione dei Bilanci e la stampa del Bullettino.

Art. 17. - Il Presidente ha di diritto l'iniziativa di tutti gli affari concernenti il buon andamento della Societa. Esso convoca e dirige le adunanze generali, e quelle deI 
Consiglio e del Comitato. Nomina all'occorrenza, Commissioni per qualche studio speciale, e legalizza colla propria firma gli Atti sociali.

Art. 18. - I Vice-Presidenti suppliscono il Presidente, che per malattia, per assenza, o per altri motivi non possa temporaneamente attendere al disimpegno delle proprie incombenze.

Art. 19. - Uno dei Segretari ha cura di tuttociò che riguarda gli Atti della Società: all'altro invece è interamente affidato il Carteggio. In caso d'imperimento, l' nno supplisce l'altro.

Art. 20. - Il Tesoriere riscuote le entrate della societì e paga sopra mandati inuniti della firma del Presidente e del Segretario degli Atti, rimanendo responsabile in proprio di tutti i pagamenti fatti senza tale formalita. Firma le ricevute per le tasse dei Soci, e quelle per l'associazione al Bullettino. Tiene in perfetta regola un libro li entrata e di uscita, e presenta a tempo debito il bilancio consuntiro e quello preventivo.

Arт. 21. - I Consiglieri coarliuvano il Presidente in tutti gli studî e proposte tendenti all'incremento della Società, e suppliscono gli altri membri del Consigtio o del ('omitato, che siano per qualche cagione impediti.

Art. 22. - Il Consigliere Archivista-Bibliotecario ha in consegua il residuo delle copie del Bullettino ed ogni altra stampa della Societá, i libri venuti in dono od in cambio, i documenti ad esso trasmessi dalla Presidenza e dai Segretari, el è incaricato della spedizione del Bullettino e degli stampati sociali di qualunque genere.

ArT. 23. - - Il Comitato residente elegge nel suo seno ogni anno i Compilatori del Bullettino.

\section{Titolo Quarto.}

\section{Delle anumanze gencmali e panziali dei soci.}

ArT. 24. - Ogni anno, in primavera si terrà un'adunanza generale privata per la discussione e l'approvazione del bilancio consuntivo dell'esercizio precedente, per le elezioni delle cariche, e per trattare qualunque altro affare interno della Società. Di questa adumanza i Soci saramno avvertiti almeno un mese prima della sua convocazione. 
Arт. 25. - Nell'occasione di che sopra si terrà un'adunanza pubblica, nella quale il Segretario degli Atti renderà conto dei lavori scientifici compiuti dalla Societi nell'anno precedente ed il Segretario del Carteggio informerà sulle relazioni sociali, parlando pure delle opere ricernte in dono od in cambio.

ARт. 26. - Alcune admanze straortinarie, una delle quali in una citta d'Italia fuori della sede della Societá, potranno essere convocate nell'amo a cura del Comitato residente.

Aвт. 27. - Ovunque si possano rimnire almeno dieci Soci, è in loro facoltà di tenere adumanze parziali, dirette al fine che la Societá si propone; avvertendo però che coloro i quali le presiedono sono in dovere di comunicare al Comitato residente il frutto dei fatti studî per essere trasmessi ai Compilatori del Bullettino.

\section{Titolo Quinto.}

\section{Del IBulletrino.}

Aлт. 28. - La Societá pubblica, come è detto agli art. 2, 20, 22 e 23, un periodico col titolo di Bullettino della Societì Entomologica Italiana. Esso racchinde seritti originali (specialmente tendenti al illustrare la fauna d'Italia), sunti di lavori nazionali e stranieri, e tuttoció che attiene agli Atti sociali. Viene in luce ogni tre mesi in fascicoli contenenti non meno ili $S 0$ pagine ciascuno, e possibilmente corredati di tavole incise e talvolta pur colorate.

Arт. 29. - Il prezzo di associazione, rappresentato clalla tassa sociale, è di lire italiane 15. Cotal somma dovrà esser pagata, da coloro che non appartengono alla Societa, appena ricevita la prima dispensa.

ARr. 30. - I fascicoli vengono speriti franchi di posta fino a domicilio.

Arт. 31. - L'intero volume annuo, le eni dispense non son poste separatamente in rendita, costa, pei non ascritti alla Società, nè associati al giornale, lire italiane 15. I vo. lumi arretrati saramo cecluti ai Soci col 30 per 100 di ribasso.

Arт. 32. - I lavori destinati al Bullettino, debbono esser composti $o$ in italiano, o in latino, o in ambedue queste 
lingue. Potranno essere accolti anche lavori scritti in tedesco, inglese o francese, purchè gli autori sieno membri della Societa.

Ars. 33. - Non sono accettati per la stampa i manoscritti privi di sufficiente chiarezza di scrittura, o che trattino argomenti che nom sieno in relazione assai stretta con l'entomologia.

Arт. 34. - La Societá concede agil autori n. ${ }^{\circ} 50$ copie a parte dei loro lavori, purchè ne facciano la opportma richiesta nell'inviare i manoscritti.

La spesa dell'incisione e dell'eventuale coloritura delle tavole concordate coi compilatori del Bullettino, sarà per metà a carico degli antori.

Qualora l'antore desideri avere più delle cinquanta copie gratuite le otterrà ai seguenti prezzi, non compresa la spesa per la tiratura e la coloritura delle tavole corrispondenti:

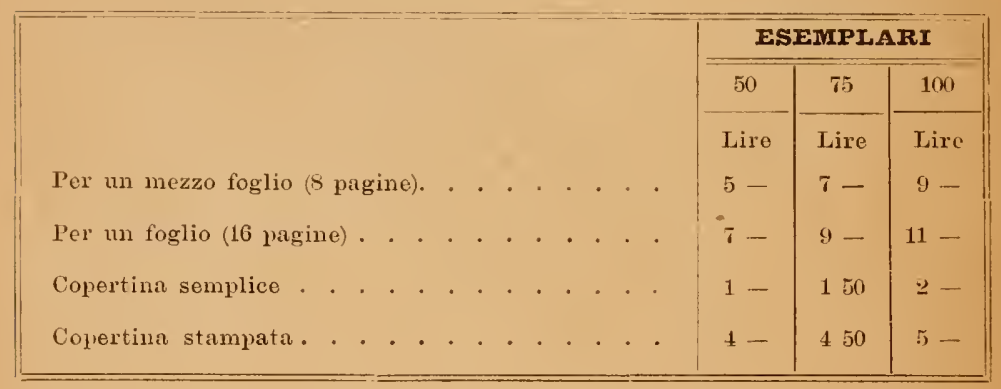

ART. 35. - Agli autori delle memorie pubblicate nel Bullettino compete interamente la responsabilità delle opinioni e dei fatti da essi esposti.

\section{Articoli trancitori.}

ART. 36. - Il Comitato residente è incaricato di provvedere ad ogni emergenza, salvo il render conto del suo operato nella successiva adumanza generale. 


\title{
CONSIGLIO GENERALE
}

\author{
DOPO LE ELEZIONI DELL'ANNO 1894
}

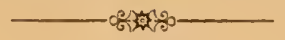

\section{Presidente.}

TARGIONI TOZZETTI Comm. Prof. Adolfo - Firenze, 1893.

Vice-Presidenti.

DORIA March. Comm. Giacomo - Genova, 1892.

PAVESI Prof. Comm. Pietro - Pavia, 1894.

STEFANELLI Cav. Prof. Pietro - Firenze, 1891.

Segretario degli Atti.

SENNA Dott. Angelo - Firenze, 1894.

Segretario delle Corrispondenze.

MARCHI Cav. Prof. Pietro - Firenze, 1893.

\section{Tesoriere.}

PASSERINI Conte NAPoleone - Firenze, 1893.

\section{Consiglieri.}

BARgagli Nob. Cav. Piero - Firenze, 1891. BAUDI DI SELVE Cav. Flaninio - Torino, 1892.

CARRUCCIO Prof. Antonio - Roma, 1892.

CATANI Dott. Prof. Tommaso - Firenze, 1894.

CAVANNA Prof. Cav. Guelfo - Roma, 1894.

COSTA Prof. Comm. Achille - Napoli, 1891.

CURÒ Ing. ANTONio - Bergamo, 1894.

EMERY Prof. CARlo - Bologna, 1891.

GESTRO Dott. RaFFaello - Genova, 1894.

GIGLIOLI Comm. Prof. Enrico H. - Firenze, 1891.

MAGRETTI Dott. PAolo - Milano, 1894.

PICCIOLI Cav. Ferdinando - Firenze, 1891.

\section{Consigliere Archivista-Bibliotecario.}

BARgagli Nob. Cav. Piero, predetto.

$$
\text { Sindaci - per l'anno } 1894 .
$$

CAROBBI Giuseppe di Giulio - Firenze.

DELla TORRE Carlo ERnesto - Firenze. 


\section{COMITATO RESIDENTE \\ DOPO LE ELEZIONI DELL'ANNO 1894

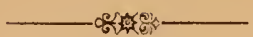

Presidente.

TARGIONI TOZZETTI Comm. Prof. Adolfo.

Vice-Presidente.

STEFANELLI Cav. Prof. Pietro.

Segretario degli Atti.

SENNA Dott. ANGELo.

Segretario del Carteggio.

MARCHI Cav. Prof. Pietro.

Tesoriere.

PASSERINI Conte Prof. NAPoleone.

Consiglieri.

BARgAGLt Nob. Cav. Pieno - Bibliotecario-Archivista. CATANI Dott. Prof. Tommaso.

GIGLIOLI Comm. Prof. Enrico H.

PICCiOli Cav, Ferdinando.

Compilatori del Bullettino.

TARGIONI TOZZETTI Comm. Prof. Adolfo. Stel'ANelli Cav. Prof. Pietro.

Bargagli Nob. Cav. Piero.

SENNA Dott. ANGELO. 


\section{ELENCO DEI SOCI}

DELLA

SOCIETA ENTOMOLOGICA ITALIANA NELL'ANNO 1894

con la indicazione della loro residenza,

degli studj da essi particolarmente coltirati, e della data della respetija elezlone

Sua Maestà UMHERTO I. Re d'Italia, - Roma. - 1873.

ALOI Prof. Antonio - Catania, R. Istituto Tecnico - Entomo. logia generale ed applicata. - 1889.

ANCONA (D') Cav. Dott. Prof. Cesare - Firenze, Piazza S. Marco, 2 - Entomologia generale - 1869.

AR'TIMINi Prof. Antonino - Firenze, Via di Soffiano, 33 - Entomologia generale - 1885.

BARGAGLI Nobile Cav. Piero - Firenze, Piazza S. Maria, Palazzo T'empi, 1 - Coleotteri, Entomologia agraria - 1869.

BARONI Giovanni - Firenze, Archivio di Stato, Via della Forca - Coleotteri e Lepidotteri - 1869.

BAUDI DI SELVE Cav. Flaninio - Torino, Via Baretti, 18 Coleotteri - 1869.

BA'TELLI Dott. Prof. Andrea - Perugia, Iibera Universita Inatomia degli Artropodi -- 1876.

BERLESE Dott. ANTonio - Portici (Napoli), R. Scuola superiore di Agricoltura - Entomologia generale ed applicata; Acari e Miriapodi - 1882.

BERTOLINI Dott. Stefano - Pergine per Madrano. ('Trentino, Impero Austro-Ungarico) - Coleotteri - 1869.

BER'TOLONI Cav. Prof. Antonio - Bologna, fuori Porta Mosco. vella - Entomologia applicata - 1881.

BEZZI Dott. Mario - I'rofessore nel R. Liceo di Macerata, Via Corso Vecchio, 104 - Entomologia generale, Ditteri, Enitteri - $1 \$ 91$. 
12

BRUSINA Prof. SpIRIdIone - Agram. (Zagabria, Croazia) - Entomologia generale - 1879 .

CALANDRUCCIO Dott. Prof. Salvatore - Catania, R. Università

- Entomologia generale ed applicata - 1889.

CALLEGARI Ferdinando - Treviso - Entomologia generale - 1871 .

CAMERANO Dott. Prof. Lorenzo - Torino, R. Museo zoologico - Entomologia generale - 1878.

CANTONI Dott. Prof. Elvezio - Milano, R. Liceo Manzoni Aracnidi e Miriapodi - 1880.

CAROBBI Giuseppe - Firenze, Via di Mezzo, 54 - Ortotteri - 1880 .

CARRUCCIO Cav. Prof. Antonio - Roma, R. Università - Entomologia generale - 1869.

CAVANNA Dott. Prof. Cav. Guelfo - Roma, R. Ministero della Pubblica Istruzione (Ispettorato) - Entomologia generale; Aracnidi, Miriapodi, Emitteri - 1875.

CATANI Dott. 'Tommaso - Firenze, Palazzo da Cepparello, Via del Corso, 4 - Entomologia generale - 1882.

CORTI Dott. EmILIo - Pavia, R. Universita - Entomologia gene. rale, Ditteri - 1894 .

CORSINI Principe Tommaso - Firenze - Entomologia applicata - 1869.

COSTA Comm. Prof. Achille - Napoli, R. Museo zoologico Entomologia generale - 1869.

CURÒ Ing. AnTonio - Bergamo - Lepidotteri - 1869.

DEI APElle - Siena, Via dei Tufi, 1 - Entomologia generale - 1869 .

DEL GUERCIO Dott. Giacoso - Firenze, R. Stazione di Entomologia agraria - Entomologia applicata; Omotteri - 1891.

DESBROCHERS DES LOGES JuLES - Tours (Indre et Loire, Francia), Rue de Boisdénier, 23 - Coleotteri paleartici; Curculionidi e Cassidi del globo - $189 \mathrm{I}$.

DE CARLINI Dott. Prof. Angelo - Pavia, R. Liceo Foscolo Entomologia generale; Emitteri, Aracnidi - 1889. 
DI FRASSINETo Conte Alfredo - Firenze, Via Palestro, 3 Entomologia generale - 1891.

I)E VINCENZI Comm. Giuseppe, Senatore del Regno - Roma Entomologia applicata - 1869.

DORIA Marchese Comm. Gucovo, Senatore del Regno - Genova, Tia Peschiera, 28 - Coleolteri - 1869.

EMERY Prof. Carlo - Bologna, R. Universita - Coleotteri curopei e mediterranei; Formicidei - 1869.

FAILLA-TEDALDI Luigi - Castelbuono Madonie, Sicilia - Entomologia generale; Lepidotleri - 1874.

FICALBI Dott. Prol. Eugenio - Cagliari, R. Università - Entomologia generale; Culicidei - 1889.

FOCARDI Lorenzo - Firenze, Via Pellicceria, 7 - Entomologia generale - 1882.

FRANCESCHINI Cav. Ferice - Milano, Via Monforte, 14 Entomologia applicata - 1890.

GASPERINI Prof. RICCARdo - Spalato (Dalmazia) - Entomologia generale, Imenotteri - 1869 .

GESTRO J)ott. Prof. Cav. Raffaello, Vice Direttore del Museo Civico di Storia Naturale di Genova - Entomologia generale; Coleotteri - 1871 .

GIANELLI Giacinto - Torino, Via Manzoni, 2 - Lepidotteri - 1869.

GIBSON CARMICHAEL 'T'HoMAS - Castleeraig, Dolphinton (Scozia) - Miriapodi - 1882.

GlGLiOLI Comm. Prof. Enrico - Firenze, R. Musco, Via Romana, 19 - Entomologia generale - 1869.

GOSS HerberT - Berrylands, Surbiton Hill, Kingstown on 'Thames, Inghilterra - Entomologia generale - 1890.

GRASSl Dott. Prof. Grovan Battis'1 - Catania, R. Universiti Entomologia generale - 1889.

GRESSEL Dott. Agostino - Trento, Via Larga, 4 - Coleotteri - 1890.

GRIBODO Ing. GIovaxnı - Torino, Via Maria Vittoria, 19 - Imenotteri -1872 . 
HEYDEN (VoN) Dott. Maggior LuCA - Bokenheim, prosso Francoforte sul Meno, Schlosstrasse, 54 - Insetti di tutti gli ordini, principalmente Coleotteri - 1870.

LEVEILlÉ Albert - Paris, Rue S. ${ }^{t}$ Placide, 42 - Entomologia generale - 1891.

LORENA (DI) Sua Altezza l'Arciduca Luigi Salvatore - Trieste per Muggia, Villa Zindis, Impero Austro-Ungarico - Entomologia generale - 1869.

LOSTIA DI SANTA SOFIA Conte Umberto -- Cagliari, Via Canelles, 15 - Entomologia generale - 1884.

MACLACHLAN Roberto - Londra, Lewisham, Westview, Clarendon Road - Nevrotteri - 1869.

MAGRETTI Dott. PAolo - Milano, Via Dante, 7 - Imenotteri -1880 .

MANTERO Giacono - Genova, Via Galata N. 9 (int. 7) - Inenotteri - 1894 (Socio studente).

MANZONE Dott. Prof. FAUstino - Brà, Pjemonte -.. Enlomoloyia generale - 1890.

MARANDINO Ovidio - Firenze, Via del Campuccio N. 4 - Entomologia generale - 1894 (Socio studente).

MARCHI Cav. Prof. Pıetro - Firenze, Via degli Alfani, 50 Entomologia generale - 1869.

MAR'TELLI Nob. Ugolino - Firenze, Via della Forca, 8 - Entomologia generale - 1879.

MELla Conte Carlo Arborio - Vercelli, Piemonte - Entomo. logia generale - 1869.

MEREGHI Dott. CARLo - Iesi (Marche) - Entomologia generale -1881 .

MICHAILOWITCH. Sua Altezza Imperiale il Granduca Nicolas MiCHAILOWitch - Pietroburgo - Lepidotteri - 1883.

OSTEN SACKEN Barone Dott. Carlo Roberto - Heidelberg Ditteri - 1880 (Socio perpetuo).

PASSERINI Conte Prof. Napoleone - Scandicci (Firenze) — Entomologia agraria - 1879.

PAVESI Prof. Comm. Pietro - Pavia, alla R. Universiti, Direttore del Museo di Zoologia - Entomologia generale; Aracnidi - 1878. 
PANATTONI Nob. Avv. Guido - Firenze, Via S. Egidio, 11 Entomologia applicata - 1892.

PECORI Rag. Raffaeldo - Firenze, Via dei Conti, 3 - Entomologia applicata - 1891.

PICCHI Luigi - Firenze, Via Pandolfini, 26 - Entomologia generale -1889 .

PICCINELLI Dott. GiovanN1 - Bergamo, Via Masini, 9 - Coleotteri -1879.

PICCIOLI Cav. Ferdinando, - Firenze, Borgo degli Albizzi, 25 -- Entomologia generale; Coleotteri e Imenotleri - 1869.

PONS Guido - Firenze, Via della Chiesa, 73 - Entomologia generale - 1894 (Socio studente).

RIDOLFI Comm. March. Luigi - Firenze, Via Maggio, 13 - Encomologia agraria - 1869.

RONCALLI Conte Dott. Alessandro - Bergamo (Alta Citti) Enlomologia generale - 1883.

ROVELLI Dott. Giuseppe - Como, Piaza Volta, 4 - Entomologia generale -1890.

SELYS-LONGCHAMPS (DE) Cav. Senator Barone Edrondo Liegi (Belgio), Boulevard de la Sauvemière, 34 - Entomologia generale; Nevrolteri - 1869.

SENNA Datt. Angelo - Firenze, R. Museo, Via Romana, 19 Entomologia generale; Brentidi - 1891.

SIMON Eugène, già Presidente della Società Entomologica di Francia - Parigi, Villa Said, 16 - Aracnidi.

STAUDINGER Dott. Otтo - Blasewitz presso Dresda (Sassonia) - Lepidotteri - 1870.

STEFANELli Cav. Prof. Pietro - Firenze, Via Pinti, 57 - Lepidotleri - 1869.

TARGIONI-TOZZETTI Comm. Dott. Adolwo, Prof. di Zoologia e Anatomia comparata nel R. Istituto di Studi Superiori - Firenze, Via Romana, 19 - Enlomologia generale ed applicata; Ortotteri, Crostacei etc. - 1869.

ThOREll Plof. 'Tamerlano - Montpellier (Herault), France Aracnidi - 1877. 
TORRE (DELLA) nob. CARLo - Firenze, Via Domenico Buonvicini, 4 - Entomologia generale e applicata: Crisidi, Icneumonidi - 1878.

TURATI Conte Emilio - Milano, Via Meravigli, 13 - Entomologia generale - 1874.

TURATI Conte Gianfranco - Milano, Via Meravigli, 7 - Lepidotteri - 1883.

VERDIANI-BANDI Dott. ARNaldo - San Quirico d'Orcia, (Provincia di Siena) - Entomologia generale - 1874.

VERDIANI-BANDI Dott. Lurai - Siena, Via Ricasoli, 52 - Coleotteri europei - 1871 .

VERRAL G. H. Esq. Sussex Lodge, Newmarket, England - Ditteri - 1881. (Socio perpetuo).

VIMERCATI Conte Prof. Ing. Guido - Firenze, Lungarno della Zecca, 2 - Entomologia generale - 1870.

\section{Soci defunti nell'anno 1893.}

MOLFino Prof. Giovan Maria - Genova, 1878.

PASSERINI Comm. Prof. Grovanni - Parma, 1869.

STAINTON H. T. - Londra, 1869.

\section{Soci dimissionari.}

BECHI Comm. Prof. Emilio - Firenze, 1869.

CASTRACANE DEGLI ANTELMINELLI ALessandro - Rimini, 1875.

TARGIONI Dott. Francesco - Firenze, 1883. 
ELENCO delle Società, Accademie ecc. che fanno cambio delle loro pubblicazioni col Bullettino della Società Entomologica Italiana.

\section{I'TALIA.}

Bolognd -- R. Aecademia delle Scienze dell' Istituto di Bolngna.

Catania - R. Accademia Gioenia di Scienze Naturali.

Firenze - Nuovo Giornale botanico, diretto dal Prof. 'T. Caruel.

Genora - Museo civico di Storia naturale; Direttore Comm. Giacomo Doria.

Milano - Società italiana di Scienze naturali.

Modena - Societi dei Naturalisti.

NAPOLt - R. Accademia delle Scienze fisiche e matematiche.

- Societil di Naturalisti. (ex Monastero della Sapienza).

PAdora - Societi veneto-trentina di Scienze naturali.

- Bollettino mensile di Bachicoltura, diretto dai Proff. Verson e Quajat.

Palervo - Il Naturalista siciliano. (Dirett. sig. E. Ragusa).

Pavia - Bollettino scientifico, diretto dai Proff. Maggi, Zoja ecc.

Roma - R. Comitato geologico d'Italia.

- R. Accademia dei Lincei.

- Accademia Pontificia dei Nuovi Lincei.

- Societi romana per gli studi zoologici.

Siexa - R. Accademia dei Fisiocritici.

- Rivista italiana di Scienze naturali e Bollettino del Naturalista. (Dirett. Cav. S. Brogi).

UdNe - Associazione agraria friulana.

Venezia - R. Istituto Teneto di Scienze, Lettere ed Arti.

\section{AUSTRIA UNGHERI..}

BRÜNN - Naturforscheude Verein.

Budarest - Királyi 'Természettudominyi 'Tà'sulat Megbizisibuil (R. Societa Ungherese di Scienze Naturali). 
BudApest - A Magyar Allatani Irodalom. (Accademia Ungherese delle Scienze.

Gorizia - Imperiale e Reale Società Agraria.

Graz - Naturwissenschaftliche Verein für Steiermark.

InNsBruck — Naturwissenschaftlich-medizinische Verein.

Kolosvar - Értesitö az erdélyi Muzeum-egylet Orvos-Termeszettudományi szakosztályábùl. (Sezione delle Scienze naturali e mediche della Societa del Museo di Transilvania:

Trencsén - Evkönyve. Jahresheft des Naturwissenschaftlichen Vereines d. 'Trencsener' Comitates.

Trieste - Societi adriatica di Scienze naturali.

- Museo civico di Storia naturale (Dirett. Plof. C. De Marcliesetti).

WIEN - Jahresbericht d Wiener Entomologischen Vereines.

- K. K. Zoolog.-botanische Gesellschaft.

- K. K. Naturhistoriches Hofmuseum (Prof. Franz v. Hauer).

- Verein zur Verbreitung naturwissenschfatliche Kenntnisse.

\section{SVIZZERA.}

BERn - Schweizerischen entomologischen Gesellschaft.

Neuchìtel - Société des Sciences naturelles.

\section{FRANCIA.}

Amens - Socicté Linneenne du Nord de la France.

CAEN - Société Linnéenne de Normandie.

Chateauroux - Le Frélon. Journal d'Entomologie. (Dirett. J. Desbrochers des Loges).

Cherburg - Société Nationale des Sciences naturelles.

Gray - (Haute Saine) - Species des Hyménopteres d'Europe etc.

(M. ${ }^{r}$ notaire Andrè). 17, Rue des Promenades.

Lyon - Socièté Linnéenne. (M.' Georg, libraire).

PArts - Feuille des Jeunes naturalistes. (Rue Pierre Charron, 35). Sociéte entomologique de France.

- Société Philomatique. (Rue des Grands Augustins, 7).

Rouen - Société des Amis des Sciences naturelles.

SÉsur -- Société des Sciences historiques et naturelles.

Tolouse - Société d'Histoire naturelle. 


\section{BELGIO.}

Bruxelles - Academie Royale des Sciences, des Lettres et des Beaux-Arts de Belgique. (Annuaire et Bulletin).

- Société Entomologique de Belgique.

- Société Malacologique de Belgique.

SPAGNA.

Madrid - Sociedad Española de Historia natural.

\section{GERMANIA.}

Augsutra - Naturwissenschaftliche Verein für Schwaben und Neuburg.

Berlin - Berliner Entomologischer Verein pr. ad. Herrn. B. Hache, Berlin IV. 64. Charlotten Strasse, 37-38.

- Deutsche Entomologischer Gesellschaft (D. ${ }^{\mathrm{r}}$ G. Kraatz).

Boxs - Natarhistorischer Verein der preuss. Rheinlande und We. stfalens.

Brdunschweig - Verein für Naturwissenschaft.

BresLau - Verein für schlesische Insectenkunde.

CASSEL - Verein für Naturkunde.

Dresden - Isis: Naturw. Gesellschaft.

- Iris: Correspondenz-Blatt des Entomologischen Vereins.

Giessen - Oberliessische Gesellschaft für Natur-und Heilkunde.

Halle - Academia Cresarea Leopoldino-Carolina Germanica Naturae curiosorum.

Heidelierg - Naturhistorisch-medicinischer Verein.

LeipZig - Zoologischer Inzeiger. (Prof. Victor Carus).

Müster - Westfalischer Provinzial Verein für Wissenschaft und Kunst. (Prof. Landois).

Mrcklasiburg -- Verein der Freunde der Naturgeschichte res. in Güstrow.

Nurveerg - Naturhistorische Gesellschaft.

Offenbach - Offenbacher Verein fül Naturkunde.

STETTin - Entomologischer Verein.

StutTGart - Verein für vaterlündische Naturkunde in Würtemberg.

IVernigerode - Naturwissenschafliche Verein des Harzes.

Wresbadex - Nassauischer Verein für Naturkunde. 
OLANIDA.

Leiden - Nederlandische entomologische Vereeniging.

\section{DANIMARCA.}

Kíobenhavn - Kongelige Tanske Videnskabernes Selskabs.

\section{SVEZIA E NORVEGIA.}

Stoскиода - Entomologiska Föreningen. (Prof. G. Hofgren).

- Kongl. Svenska Vetenskaps-Akademie.

\section{GRAN BRETTAGNA ED IRLANDA.}

BeLfast (Ireland) - Natural History and Philosophical Society.

Grasgow (Scotland) - Society of Natural History.

London (Ergland) - Entomological Society.

- - Royal Microscopical Society.

- - The Entomologist, an illustrated Journal of Britislı entomology.

- _ The Entomologist's Montlıly Magazine.

\section{RUSSIA E FINLANIDIA.}

КнARKоFF - Societé des naturalistes à l'Universitè Imperiale. (in russo).

Mosca - Société Imperiale des Naturalistes.

- Societa Imperiale degli amici delle Scienze naturali ecc. aggiunta all'Università di Mosca. (in russo).

Odessa - Societa dei naturalisti della Nuova Russia. (in russo).

Pietroburgo - Horre Societatis entomologicæ rossicre.

Helsingfors - Societas pro Fauna et Flora Fennica. (Atti e Rendiconti).

\section{STATI UNITI.}

Albany - New York State Museum of Natural History.

BaLtimora - Johns Hoplins University.

Boston - Society of Natural History.

BuffaLo - Society of Natural Sciences.

Canbridge (Mass) - Cambridge entomological Club-Psyche.

Chapel Hill (N. Carolina). - Elisha Mitchell Scientific Society. 
Filadelfia - American Entomological Society.

- Academy of Natural Sciences (Nolan I. Edw. Bibliot.)

- Wagner free Institute of Sciences.

LAWrence - 'The Kiansas University Quarterly.

MADISOx - Irisconsin Academy of Sciences.

Mrnveapolis - The University of Minnesota (F. B. Gilfillan Secret.) SALEM - Essex Institute.

San Francisco - California Academy of Sciences.

'Toperi - Kansas Academy of Science.

Trenton - N. Jersey Natural History Society.

WASHINGToN - Department of Agriculture.

- Entomological Society of IVashington.

- Smithsonian Institution.

- United States Geological Survey. Department of the Interior.

\section{CANADA.}

OtтаWA - Geological and Natural History Survey of Canada. (Alfred Selwyn Director).

Toronto - Canadian Institute.

\section{AMERICA CENTRALE E MERIDIONALE.}

Brasile - Museu Nacional in Rio de Janeiro (Sen. Ladislau Netto). MEssico - Sociedad cientifica Antonio Alzate in Mexico.

Repubbica Argentina - Sociedad cientifica Argentina in Buenos Ayres.

SAy Josè (Costa Rica) - Museo Nacional.

\section{AUSTRALIA.}

Adelaide - Royal Society of South Australia.

Melbourne - Public Library, Museum and National Gallery, Victoria. SrDNEY - R. Society of the New South Wales.

TASMania - Royal Society of Tasmania. 


\title{
BLENCO DEGLI ASSOCATTI AL BLLLETTINO
}

\author{
Stazione agraria di Palermo.* \\ $\gg \quad \gg$ Torino.* \\ $\gg \quad$ »Modena.* \\ ๖ bacologica di Padova." \\ » di Botanica crittogamica di Pavia.* \\ Scuola superiole di agricoltura in Portici (Napoli).* \\ » di agricultura in Milano.* \\ » di Viticoltura e di Enologia di Catania.." \\ » enologica in Avellino.* \\ $\gg$ pratica di Agricoltura in Scerni (Chieti).* \\ $\gg \gg$ in Caluso (Torino).* \\ $\gg \gg$ in Catanzaro.* \\ » $\gg$ in Pozzuolo nel Friuli (Udine).* \\ $\gg$ » $\gg$ in Caltagirone.* \\ » $\gg$ in Cesena (Provincia di Forli)." \\ » di Agricoltura ed Enologia in Conegliano (Treviso)." \\ »enologica di Alba.* \\ » di Pomologia in Firenze.* \\ Ministero Agr. Ind. e Com., Roma** \\ R. Istituto forestale di Tallombrosa."
}

N.B. Lasterisco indica gli associati per conto del R. Ministero di Agricoltura Industria e Commercio.

Friedländer R. e figlio N. IV. Berlino, Carlstrasse, 11 (5 copie). Loescher Ermanno Libraio-Editore, Tia Po, 19, Torino (5 copie). Ongania Ferdinando Succ. Munster, Libraio-Editore. Venezia. (Per la Biblioteca di Monaco).

Museo di Storia Naturale della R. Università di Modena. 
Nuseo civico di Storia Naturale nel R. Istituto tecnico Antonio Bordoni, Pavia.

Bibblioteca del R. Istituto 'Tecnico in Firenze.

$\checkmark \quad$ del R. Liceo Dante in Firenze.

» del R. Museo di Fisica e Storia Naturale, Gabinetto Invertebrati. Firenze.

Nazionale di Parma.

» della R. Università di Genova.

$\gg$ della R. Universitá di Torino.

Il Bullettino viene mandato in dono al R. Ministero della Pubblica Istruzione, in Roma; alla Stazione zoologica Dohrn in Napoli, ed al Sir. Dottore Adollo Senoner, Landstrasse, Krieglergass:, 14, Vienna. 


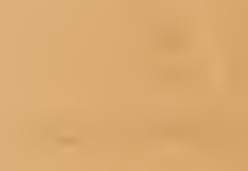

( 



\section{PAMPHLET BINDERS}

This is No. 1524 atar carried In stock in the falsowing alree MIGH wiDe TMICXress

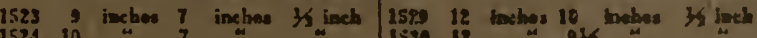

1524

1523

1526

1587

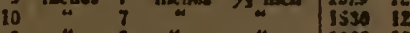

a 1932 11

* $10^{\circ}$ a

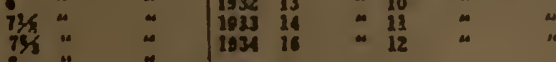

Other sizos madi to order.

\section{MANUTACTUAED BY}

LIBRARY BUREAU

Division of REMINGTON RAND INC.

Lobrary Supplias of all Kiscis 


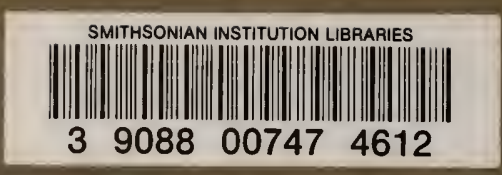

